\title{
Standounkt
}

Anpassung und Nachhaltigkeit

\section{Synonym, Antonym oder etwas dazwischen?}

Klimaanpassung ist eine komplexe Herausforderung für die Gesellschaft und ihre Akteure. Mit Nachhaltigkeit soll „(...) etwas bewahrt werden zum Wohl der zukünftigen Generationen“, so Bernd Klauer. Gleiches gilt für die Anpassung an den Klimawandel und trotz dieser ähnlichen Ziele wird bisher in diesem Rahmen zu wenig auf Nachhaltigkeit geachtet.

Von Kevin Grecksch und Maik Winges

B eginnen wir mit der Frage, welche Prozesse und Zustände angepasst werden. Zugrunde liegt dabei zu oft eine Extrapolationslogik: Wir machen uns auf den Weg, ohne zu wissen wohin, und gehen daher alte Pfade weiter, ohne zu fragen, ob diese überhaupt wünschenswert sind. Zum Beispiel: Sollen wir uns wieder mehr zersiedeln, um Hitzeinseln in hoch verdichteten Ballungszentren $\mathrm{zu}$ vermeiden?

Die gewünschte Zukunft muss auf Basis einer breiten gesellschaftlichen Debatte formuliert werden. Nachhaltige Entwicklung ist ein - wenn auch unterschiedlich interpretiertes Ziel - welches mittlerweile in der deutschen Gesellschaft breite Zustimmung findet (BMU/ UBA 2011).

\section{Soziale Auswirkungen...}

Bei der Berücksichtigung von Nachhaltigkeit in der Anpassung wird häufig vordergründig die ökonomische Säule berührt. Wirtschaftliche Risiken bändigen und sich eröffnende Chancen nutzen. In vielen Fällen wird eine ökologische Komponente betrachtet, etwa dann, wenn technische Lösungen auf ihre Wirkungen auf Natur und Klima hin untersucht werden.

Es besteht weitgehende Einigkeit darüber, keine Anpassung auf Kosten der Mitigation zu betreiben. Neben anderen blinden Flecken werden insbesondere die sozialen Auswirkungen des Klimawandels noch nicht ausreichend betrachtet. Ebenso wie Regionen sind auch soziale Grup- pen wie Senioren, Migranten oder Kranke unterschiedlich stark vom Klimawandel betroffen. Der Hitzesommer 2003 und der Hurrikan Katrina haben uns das vor Augen geführt; bisher wurden aber nur unzureichende Konsequenzen gezogen.

\section{... von Maßnahmen der Anpassung}

So wird auf lokaler Ebene fleißig partizipiert, nicht immer jedoch mit befriedigenden Ergebnissen. Prozesse können verlangsamt, delegitimiert oder durch ressourcenstarke Akteure beeinflusst werden. Auf Ebenen, in denen eine umfassende Beteiligung nicht möglich ist, werden Interessen benachteiligter Gruppen weniger berücksichtigt oder es kommt zu technischen Fallstricken, wie beim $\mathrm{Zu}$ kunftsdialog der Bundesregierung (Beuth 2012).

Genauso wie Extremereignisse werden Anpassungsmaßnahmen Verteilungswirkungen haben. Das betrifft auch die übergeordnete Rahmensetzung insbesondere vor dem Hintergrund der in den letzten Jahren häufiger geforderten Eigenvorsorge. Selbstverständlich ist es sinnvoll, darüber nachzudenken, wie riskantes Verhalten bei gleichzeitigem Verlass auf den Staat verhindert werden kann. Ein Beispiel: Es sollten keine Anreize bestehen, in überschwemmungsgefährdeten Gebieten zu siedeln und im Schadensfall staatliche Hilfe $\mathrm{zu}$ fordern. Wo bereits unterschiedliche Anfälligkeiten bestehen, werden zur sozialen Abfederung öffentlich- private Versicherungslösungen, etwa finanziert durch Anpassungsfonds, angedacht. Dennoch sind letztlich die lokalen Verteilungswirkungen und die damit einhergehenden individuellen Betroffenheiten bestimmter Bevölkerungsgruppen noch nicht ausreichend klar. Dies trifft auch auf die ökonomische Fähigkeit zu, (bezuschusste) Versicherungen abzuschließen.

Dazu passt, dass die Bundesregierung im Februar 2012 unauffällig und nahezu unbemerkt mit dem „Fortschrittsbericht 2012“ ihre Bilanz nach zehn Jahren Nachhaltigkeitsstrategie präsentierte. Während die ökonomische Seite gut wegkommt, es bei der ökologischen Dimension gewaltig hapert, ergibt sich bei der sozialen Dimension ein gemischtes Bild. Die Bevölkerung wird älter, dicker und die Lohnunterschiede zwischen Frauen und Männern sind noch immer frappierend (Pötter 2011). Um nicht weitere gesellschaftliche Probleme zu schaffen oder vorhandene weiter $\mathrm{zu}$ verschärfen, muss die gesellschaftliche Herausforderung der Anpassung an den Klimawandel Nachhaltigkeit in den Blick nehmen. Und zwar nicht weniger, sondern mehr als bisher.

\section{Literatur}

Beuth, P.: Zukunftsdialog. Bürgerbeteiligung im Netz mit fragwürdigen Ergebnissen. Zeit Online vom 13.04.2012.

BMU / UBA (Hrsg.): Umweltbewusstsein in Deutschland 2010. Berlin 2011.

Klauer, B: Was ist Nachhaltigkeit und wie kann man eine nachhaltige Entwicklung erreichen? Zeitschrift für angewandte Umweltforschung, Heft 12, 1/1999: S. 86 - 97.

Pötter, B: Wieder mal Regen. tageszeitung vom 17.04.2012

I AUTOREN + KONTAKT

Kevin Grecksch und Maik Winges sind wissenschaftliche Mitarbeiter im Projekt „nordwest2050“ an der Carl von Ossietzky Universität Oldenburg.

Carl von Ossietzky Universität Oldenburg Ammerländer Heerstraße 114-118 26129 Oldenburg. Tel.: +49 4417984088 .

E-Mail: kevin.grecksch@uni-oldenburg.de; maik.winges@uni-oldenburg.de 


\section{Lizenzhinweis}

Die Beiträge in ÖkologischesWirtschaften werden unter der Creative-Commons-Lizenz "CC 4.0 Attribution Non-Commercial No Derivatives" veröffentlicht. Im Rahmen dieser Lizenz muss der Autor/Urheber stets genannt werden, das Werk darf nicht bearbeitet, abgewandelt oder in anderer Weise verändert und außerdem nicht kommerziell genutzt werden. Die digitale Version des Artikels bleibt für zwei Jahre Abonnent/innen vorbehalten und ist danach im Open Access verfügbar. 